IUHET-391

May, 1998

\title{
Are there quasistable strange baryons with anticharm or antibeauty?
}

\author{
D. B. Lichtenberg ${ }^{a}$ \\ Physics Department, Indiana University, Bloomington, IN 47405, USA
}

\begin{abstract}
In some models, exotic baryons with strangeness and anticharm or antibeauty should exist and even be stable against strong decay. We consider the stability of such possible exotic baryons, which in the constituent quark picture are called pentaquarks (each is composed of four quarks and an antiquark). Our model is based on diquark clustering and supersymmetry in hadrons, and assumes that the spin-dependent force between quarks arises from one-gluon exchange. In the model, a pentaquark with strangeness and anticharm can decay strongly, but an analogous pentaquark with an antibeauty quark is stable except for weak decay.
\end{abstract}

${ }^{a}$ lichten@indiana.edu

\section{Introduction}

Some years ago Lipkin [1] and Gignoux et al. [2] suggested that exotic baryons containing strangeness and anticharm or antibeauty might be stable against strong decay. We denote these so-called pentaquarks by $P(q q q s \bar{c})$ and $P(q q q s \bar{b})$ respectively, where $q$ denotes a $u$ or $d$ quark. So far, such exotic baryons have not been observed.

Recently, Roncaglia, Predazzi, and I [3] showed that a model of hadrons with diquark clustering, supersymmetry, and chromomagnetic spin-dependent forces as ingredients could be used to obtain the masses of some exotic hadrons in terms of the masses of ordinary mesons and baryons. The advantage of the model is that all parameters are obtained from the meson and baryon sectors - the exotic hadron masses are obtained without any additional adjustable parameters. The purpose of this paper is to apply the model to the lowest-mass (ground-state) pentaquarks of the type $P(q q q s \bar{c})$ and $P(q q q s \bar{b})$. (A strange quark has strangeness $S=-1$, a charmed quark has charm $C=1$, and a beauty quark has beauty $B=-1$; antiquarks have the opposite quantum numbers. By anticharm we mean $C=-1$, but by antibeauty we mean $B=1$.)

Various ingredients of the model have been developed in a number of papers. In Section 2 we summarize the model and give references to papers where further details may be obtained. In Section 
3 we apply the model to the pentaquarks $P(q q q s \bar{c})$ and $P(q q q s \bar{b})$. We find that the pentaquark with anticharm is unstable against strong decay into a nucleon plus a $D_{s}$ meson, whereas the pentaquark with antibeauty is stable against strong decay. It is interesting that application of heavy quark effective theory [4] to leading order (neglecting terms that go inversely with the heavy quark mass) gives either the prediction that these pentaquarks are stable or that both are unstable. We discuss why our model gives a different result, and we explain why we think that heavy quark effective theory is not applicable. Finally, we discuss some of the possible weak decay modes of the pentaquark with antibeauty.

\section{Outline of the model}

In this section, we summarize the basic ingredients of a model incorporating diquark clustering, hadron supersymmetry, and spin-dependent forces arising from one-gluon exchange. In any hadron containing at least three quarks (including antiquarks), we consider its constituent quarks as far as possible in pairs, which we call diquarks. (A quark and an antiquark is not a diquark.) For example, a baryon composed of three quarks is considered as a bound state of a quark and a diquark, an exotic four-quark meson (a tetraquark) is considered as a bound state of a diquark and antidiquark, a pentaquark is a bound state of two diquarks and an antiquark, etc. A review of diquark models has been given by Anselmino et al. [5].

Hadron supersymmetry is an approximate consequence of QCD. According to QCD, the interaction between two coloured particles depends primarily on their individual colours and on their colour configuration. We now note that a diquark belongs either to a $\mathbf{6}$ or to a $\overline{\mathbf{3}}$ representation of colour-SU(3). The $\overline{\mathbf{3}}$ is the same representation as that of an antiquark. Therefore, the transformation of replacing an antiquark (a fermion) by a $\overline{\mathbf{3}}$ diquark (a boson) in a hadron should to first approximation leave the interaction energy unchanged. This is what we mean by hadron supersymmetry. The mathematical underpinnings of the idea can be found in papers of Miyazawa [6] and Catto and Gürsey [7].

A limitation of hadron supersymmetry is that although the transformation is an approximate invariant for $\overline{\mathbf{3}}$ diquarks, the interactions of sextet diquarks are not related to those of antiquarks in the model. However, in lowest-order perturbation theory, the interaction of two quarks in a colour- $\overline{\mathbf{3}}$ state is attractive, while in a colour-6 state the interaction is repulsive. This fact leads us to conjecture that sextet diquarks have higher masses than antitriplet diquarks with the same quark content, so that if we are considering low-mass states we can safely neglect configurations containing sextet diquarks.

The supersymmetry is broken by mass, spin, and size differences between an antiquark and a diquark. Noting that neither a constituent quark nor a diquark is a point particle, we neglect the size effect. However, we are able to make approximate corrections for differences in mass and spin. Let us first consider spin.

We approximately remove the effect of spin-dependent forces by averaging over spins of mesons and baryons. We confine ourselves to ground states, and and confine our analysis to states with zero orbital angular momentum in the nonrelativistic approximation. The spin-averaged mass $\mathcal{M}$ of a vector meson $M_{1}$ and pseudoscalar meson $M_{0}$ is given by [8]

$$
\mathcal{M}=\left(3 M_{1}+M_{0}\right) / 4
$$

We can use this simple formula to obtain the value of $\mathcal{M}$ when $M_{1}$ and $M_{0}$ are known from experiment [9] In cases in which data are lacking, we can obtain estimates by interpolation or extrapolation, making use of empirical regularities in hadron masses $[10,11]$. The meson interaction energy $E_{\mathcal{M}}$ is defined in terms of the mass $\mathcal{M}$ and the constituent quark masses $m_{1}$ and $m_{2}$ :

$$
E_{\mathcal{M}}=\mathcal{M}-m_{1}-m_{2}
$$


We denote the spin-dependent part of the interaction energy between quark and antiquark by $\epsilon$, given by

$$
\epsilon=\left(M_{1}-M_{0}\right) / 4
$$

Then

$$
M_{1}=\mathcal{M}+\epsilon, \quad M_{0}=\mathcal{M}-3 \epsilon .
$$

For mesons, this procedure is straightforward, but for baryons we need a model to extract the spin-dependent interaction energy between two quarks from baryon mass differences, given that a baryon contains three quarks. We assume that the spin-spin interaction is the chromomagnetic interaction arising from one-gluon exchange. A different assumption will in general give different answers. In order to write down results, we have to order the three quarks in a baryon. We adopt the following ordering: if all three quarks have different flavours, we order them from lightest to heaviest; if any two have the same flavour, they are the first two [8]. In the case in which all three quarks have different flavours, there are three ground-state baryons, which all have zero orbital angular momentum but differ in their spin configurations. Spin-dependent forces break the degeneracy. We denote by $A$ and $A^{\prime}$ the baryons of spin $1 / 2$, which differ according to whether the first two quarks have spin zero or one. We denote by $A^{*}$ the baryon of spin 3/2. (In the case of baryons containing two $q$ quarks and an $s$ quark, $A$ is $\Lambda, A^{\prime}$ is $\Sigma$, and $A^{*}$ is $\Sigma^{*}$.) Then the spin-averaged mass $\mathcal{A}$ and interaction energy $E_{\mathcal{A}}$ are given by [8]

$$
\begin{aligned}
\mathcal{A} & =\left(2 A^{*}+A+A^{\prime}\right) / 4, \\
E_{\mathcal{A}} & =\mathcal{A}-m_{1}-m_{2}-m_{3} .
\end{aligned}
$$

We let $\epsilon_{12}, \epsilon_{13}$, and $\epsilon_{23}$ be given by

$$
\epsilon_{12}=\left(2 A^{*}+A^{\prime}-3 A\right) / 12, \quad \epsilon_{13}+\epsilon_{23}=\left(A^{*}-A^{\prime}\right) / 3 .
$$

Then

$$
A^{*}=\mathcal{A}+\epsilon_{12}+\epsilon_{13}+\epsilon_{23}, \quad A^{\prime}=\mathcal{A}+\epsilon_{12}-2 \epsilon_{13}-2 \epsilon_{23}, \quad A=\mathcal{A}-3 \epsilon_{12} .
$$

The values of $\epsilon_{i j}$ turn out to depend weakly on the mass of the "spectator" quark $k$.

If any two quarks have the same flavour, the baryon $A$ is absent because of the Pauli principle, while if all three quarks have the same flavour, both $A$ and $A^{\prime}$ are absent. We see from Eq. (8) that in these cases we cannot in general determine $\mathcal{A}$ from the baryon masses by spin averaging. However, we can estimate the values of $\epsilon_{i j}$ from empirical regularities [10,11], and then obtain $\mathcal{A}$ from Eq. (8).

We can obtain some of the values of $\mathcal{M}$ and $\mathcal{A}$ in terms of the masses of observed mesons and baryons [9], and we can determine the others with the help of observed regularities in hadron masses $[10,11]$.

The constituent quark masses in Eqs. (2) and (6) are not completely arbitrary, but satisfy certain constraints [11, 12]. For definiteness, we use the values obtained in Ref. [12]. They are (in $\mathrm{MeV})$

$$
m_{u}=m_{d}=300, \quad m_{s}=475, \quad m_{c}=1640, \quad m_{b}=4985 .
$$

It is important to note that these quark masses were obtained using as input only normal meson and baryon masses. No input from exotic hadrons was used.

Using these quark masses, we can determine $E_{\mathcal{M}}$ and $E_{\mathcal{A}}$ from the values of $\mathcal{M}$ and $\mathcal{A}$. If we now plot $E_{\mathcal{M}}$ as a function of the reduced mass $\mu$ of its constituent quarks, we find the function is rather smooth and suitable for interpolation. Because of supersymmetry, if we can estimate the mass of a diquark, we can regard it as a fictitious antiquark of the same mass, and find the interaction energy from the meson curve of $E_{\mathcal{M}}$ vs the reduced mass $\mu$.

We estimate the diquark masses as follows: We choose the two heaviest quarks in a given baryon to be the diquark. We guess that its mass is the sum of its constituent masses, calculate the reduced 
mass of diquark and the third quark, and read off the interaction energy from the curve for mesons. Adding this interaction energy to the mass of the quark and diquark will give a mass which will not in general have the value $\mathcal{A}$. However, we then vary the diquark mass until the baryon mass obtained as the sum of quark and diquark masses plus the interaction energy from the meson curve gives the value $\mathcal{A}$. This diquark mass $\mathcal{D}$ is the spin-averaged diquark mass. Then from the formula

$$
E_{\mathcal{D}}=\mathcal{D}-m_{i}-m_{j}
$$

we obtain the interaction energy of of two colour-triplet particles to form a colour antitriplet. Again it turns out that the values of $E_{\mathcal{D}}$ are rather smooth functions of $\mu$.

We next assume that the spin-dependent force between two quarks in a diquark is the same as the value we found for the diquark in a baryon. (We must neglect the weak dependence on the spectator quark.) Then we obtain for the masses of spin-one and spin-zero diquarks containing quarks $i$ and $j$

$$
D_{1}=\mathcal{D}+\epsilon_{i j}, \quad D_{0}=\mathcal{D}-3 \epsilon_{i j} .
$$

The values of $D_{0}$ and $D_{1}$ are given in Table 1 of Ref. [3].

\section{Results and discussion}

Let us first consider the pentaquark $P(q q q s \bar{c})$, which in our model contains diquarks made of $q q$ and $q s$. Because we are interested in the pentaquark of lowest mass, we choose both these diquarks to have spin zero, as the chromomagnetic interaction causes spin-zero diquarks to have lower mass than those of spin one. Note that the $q q$ diquark must be $u d$ to satisfy the Pauli principle. We neglect the Pauli principle for identical quarks in different diquarks, just as in nuclear physics the Pauli principle is usually neglected for quarks in different nucleons. This approximation is probably better for nucleons than for diquarks. A brief discussion of this approximation is given in Ref. [3].

From Ref. [3] the $q q$ and $q s$ diquarks have masses given by (in $\mathrm{MeV}$ ):

$$
D(q q)=595, \quad D(q s)=835 .
$$

Because there are no spin-dependent forces between spin-0 particles, we can directly read off from a curve giving the interaction energy of two bound triplets as a function of $\mu$ that this interaction energy $E_{Q}(Q$ stands for quadriquark) is

$$
E_{Q}=170 \mathrm{MeV}
$$

We then obtain that the spin-zero quadriquark mass $Q$ is

$$
Q=1600 \mathrm{MeV}
$$

We calculate the reduced mass of the colour triplet (the quadriquark) and the colour antitriplet (the $\bar{c}$ quark), and then read off the interaction energy from the meson curve giving the energy vs reduced mass. We obtain

$$
\mu=810 \mathrm{MeV}, \quad E_{P}=-210 \mathrm{MeV} .
$$

Adding the masses of the quadriquark and charmed quark to the value of $E_{P}$, we obtain that the mass of the penatquark is

$$
P(q q q s \bar{c})=3030 \mathrm{MeV} .
$$

We see that with this mass the anticharmed pentaquark can decay strongly as follows:

$$
P(q q q s \bar{c}) \rightarrow N(938)+\bar{D}_{s}(1969)+123 \mathrm{MeV}
$$


If we go through the same steps for the pentaquark $P(q q q s \bar{b})$, we find that the reduced mass and interaction energy are

$$
\mu=1210 \mathrm{MeV}, \quad E_{P}=-320 \mathrm{MeV} .
$$

This leads to a mass value

$$
P(q q q s \bar{b})=6265 \mathrm{MeV} \text {. }
$$

But the lowest mass state of a normal meson and baryon with the same quantum numbers as the $P(q q q s \bar{b})$ is $N(938)+B_{s}(5369)$, which has a total mass of $6307 \mathrm{MeV}$, a value greater than the mass of the $P(q q q s \bar{b})$. Consequently, in our model the pentaquark with an antibeauty quark cannot decay strongly.

By construction, these ground-state pentaquarks have no orbital angular momenta. It follows that each has spin-parity $J^{P}=\frac{1}{2}^{-}$(the negative parity arises from the fact that an antiquark has negative intrinsic parity).

Let us apply heavy quark effective theory [4] in leading order to Eq. (17) by replacing the $\bar{c}$ quark with a $\bar{b}$ quark. Then the $P(q q q s \bar{c})$ becomes $P(q q q s \bar{b})$ and $\bar{D}_{s}(\bar{c} s)$ becomes $B_{s}(\bar{b} s)$. But according to heavy quark effective theory (neglecting terms in $1 / m_{c}$ and $1 / m_{b}$ ), the hadron masses satisfy

$$
P(q q q s \bar{b})-P(q q q s \bar{c}) \simeq B_{s}-D_{s} \simeq m_{b}-m_{c},
$$

so that Eq. (17) becomes $P(q q q s \bar{b}) \rightarrow N(938)+\bar{B}_{s}(5369)+123 \mathrm{MeV}$. From these numbers, the mass of $P(q q q s \bar{b})$ is $6430 \mathrm{MeV}$, rather than our result of $6265 \mathrm{MeV}$. The difference between the two mass predictions is only $165 \mathrm{MeV}$, or about $2.6 \%$ of the $P(q q q s \bar{b})$ mass. Of course, this prediction of the mass of the antibeauty pentaquark from heavy quark effective theory depends on our result for the mass of $P(q q q s \bar{c})$. Without that input, we obtain only Eq. (20) from heavy quark effective theory.

Wherein lies the difference between the predictions of our model and those of heavy quark effective theory in leading order? According to the latter theory, both the $c$ and $b$ quarks are considered infinitely heavy compared to the masses of the light quarks. This might be a good approximation for the $D_{s}$ meson, since the $1640 \mathrm{MeV}$ mass of the $c$ quark is considerably greater than the $475 \mathrm{MeV}$ mass of the $s$ quark. However, the approximation breaks down for the $P(q q q s \bar{c})$ because even though the mass of the $\bar{c}$ quark is much greater than the mass of any of the other quarks in this hadron, the $\bar{c}$-quark mass is comparable to the sum of the masses of the light quarks, i.e., to the mass of the quadriquark $Q=q q q s$. As a result, the reduced mass of the quadriquark and $\bar{c}$ quark is considerably less than the mass of the quadriquark. On the other hand, the $\bar{b}$ quark is much heavier than the quadriquark so that the reduced mass of those two particles is not much smaller than the quadriquark mass. Because the interaction energy decreases as the value of $\mu$ increases [11], the $\bar{b}$ pentaquark is more tightly bound than the $\bar{c}$ pentaquark, resulting in the former being stable against strong decay.

If the antibeauty pentaquark decays weakly, its lifetime should be similar to the lifetimes of observed $b$-quark hadrons, or 1 to $1.5 \times 10^{-12} \mathrm{~s}$. It comes in two charge states,

$$
P^{+}=P(u u d s \bar{b}), \quad P^{0}=P(u d d s \bar{b})
$$

Decay modes should include one hadron containing a $\bar{c}$ quark. Prominent decay modes should include

$$
\begin{gathered}
P \rightarrow N+\bar{D}_{s} \text { (+ pions), } \\
P \rightarrow \Lambda \text { (or } \Sigma)+\bar{D} \text { (+ pions }), \\
P \rightarrow N+\bar{K}+\bar{D} \text { (+ pions) }
\end{gathered}
$$

There should also be decays with both hadrons and leptons in the final state.

It is interesting that in the model the $P^{0}$ has a possible two-body decay mode into a nucleon and a meson:

$$
P^{0} \rightarrow p+\bar{D}_{s}
$$


whereas the simplest two-body decay mode of the $P^{+}$leads to a $\Sigma^{+}$baryon:

$$
P^{+} \rightarrow \Sigma^{+}+\bar{D}^{0}
$$

However, we expect that most of the decays will be into multibody final states.

In our model, the pentaquark $P(q q q c \bar{s})$ has a higher mass than $P(q q q s \bar{c})$. Furthermore, the lowest-mass state with the same quantum numbers as $P(q q q c \bar{s})$ is $\Lambda_{c}(2285)+K(496)$, considerably less massive than the state $N(938)+\bar{D}_{s}(1969)$ into which the $P(q q q s \bar{c})$ can decay. Consequently, we predict that the $P(q q q c \bar{s})$ will have a large decay width, which, together with a small production cross section, will make it unlikely to be observed. For similar reasons we predict that $P(q q q b \bar{s})$ will not be observed.

We emphasize that our model depends on three ingredients: diquark clustering, hadron supersymmetry, and spin-dependent forces arising from the chromomagnetic interaction of one-gluon exchange. With these simple inputs, we have been able to calculate the masses of exotic baryons with no adjustable parameters - all parameters having been fixed from the observed properties of ordinary mesons and baryons.

But of course, there are limitations to the model. It is possible that the actual structure of exotic hadrons is different from the one we have assumed. Furthermore, if diquark clustering is important, a small admixture of colour-sextet diquarks might lower the pentaquark masses. Also, the spin-dependent forces might arise from a mechanism other than from chromomagnetism. For example, it has been proposed [13] that the spin-dependent interaction among light quarks arises from the breaking of chiral symmetry by one-boson exchange. Such a mechanism would lead to a quantitative change in our results and might also alter our qualitative conclusions.

In our approach we do not use any explicit Hamiltonian. Recently, pentaquarks have been considered in a conventional Hamiltonian model [14, 15], with spin-dependent forces arising from chiral symmetry breaking. The Hamiltonian model is quite different from our picture, and leads to different results.

Of course, it is only experiment that can distinguish between models. Therefore, experimentalists should search for both the anticharmed pentaquark and for the pentaquark with antibeauty. Moinester et al. [16] have discussed ways to look for the former. A search for the latter will be much more difficult, but in view of our prediction that it is quasistable, a serious effort to find it should be undertaken.

\section{Acknowledgments}

I should like to thank Steven Gottlieb, Renato Roncaglia, and Floarea Stancu for helpful discussions. This work was supported in part by the U.S. Department of Energy. 


\section{References}

[1] Lipkin H J 1987 Phys. Lett. B195 484 Lipkin H J 1988 Nucl. Phys. A475 307c

[2] Gignoux C, Silvestre-Brac B and Richard J M 1987 Phys. Lett. B193

[3] Lichtenberg D B, Roncaglia R and Predazzi E 1997 J. Phys. G: Nucl. Part. Phys. 23865

[4] Isgur N and Wise M B 1989 Phys. Lett. B232 113

[5] M Anselmino M, Predazzi E, Ekelin S, Fredriksson S and Lichtenberg D B 1993 Rev. Mod. Phys. 65 1199

[6] Miyazawa H 1966 Prog. Theor. Phys. 361266

Miyazawa H 1968 Phys. Rev. 1701586

[7] Catto S and Gürsey F 1985 Nuovo Cimento 86201

Catto S and Gürsey F 1988 Nuovo Cimento 99685

[8] Anselmino M, Lichtenberg D B and Predazzi E 1990 Z. Phys. C 48605

[9] Particle Data Group: Barnett R M et al. 1996, Phys. Rev. D 541

[10] Song X 1989 Phys. Rev. D 403655

Wang Y and Lichtenberg D B 1990 Phys. Rev. D 422404

[11] Roncaglia R, Dzierba A R, Lichtenberg D B, and Predazzi E 1995, Phys. Rev. D 511248

[12] Roncaglia R, Lichtenberg D B, and Predazzi E 1995 Phys. Rev. D 521722

[13] Glozman L Ya and Riska D O 1996 Phys. Rep. 268264

[14] Genovese M, Richard J-M, Stancu Fl and Pepin S, e-print hep-ph/9712452, Phys. Lett. B, to be published

[15] Stancu Fl, unpublished e-print

[16] Moinester M A, Ashery D, Landsberg L G and Lipkin H J 1996 Zeit. Phys. A 356207 